\title{
Nature-inspired learning and adaptive systems
}

\author{
Bogdan Gabrys · Davide Anguita
}

Published online: 11 February 2009

(C) Springer Science+Business Media B.V. 2009

\section{Guest editorial introduction}

Nature has been a continuous source of inspiration for many successful techniques, algorithms and computational metaphors. In this special issue, organised as part of the activities of the Nature-inspired Data Technology (NiDT) focus group within the European Co-ordinated Action project on Nature-inspired Smart Information Systems (NiSIS), we were seeking original contributions within an area of Nature-inspired technologies for Learning and Adaptive Systems. In the accepted comprehensive contributions this issue covers four quite distinctive, emerging areas inspired by broadly perceived nature and observed mechanisms and behaviours of natural systems.

In the first of these contributions Kasabov discusses a new trend currently emerging from the research into extending the well established connectionist systems paradigm. As argued by the author, in contrast to a single brain-like connectionist principle of information processing exploited in the current connectionist systems, the proposed integrative connectionist learning systems (ICOS) integrate in their structure and learning algorithms principles from different hierarchical levels of information processing in the brain, including cognitive-, genetic- and quantum-levels. The author further postulates that ICOS can be used to solve more efficiently challenging biological and engineering problems when fast adaptive learning systems are needed to incrementally learn in a large dimensional space.

While biological metaphors and inspirations coming from the attempts to understand and model information processing, adaptation and learning mechanisms which can be

B. Gabrys $(\bowtie)$

Smart Technology Research Centre, Computational Intelligence Research Group, School of Design,

Engineering and Computing, Bournemouth University, Bournemouth, UK

e-mail: bgabrys@bournemouth.ac.uk

URL: http://dec.bournemouth.ac.uk/staff/bgabrys/

D. Anguita

DIBE - University of Genoa, via Opera Pia 11A, 16145 Genoa, Italy

e-mail: anguita@dibe.unige.it

URL: http://www.smartlab.dibe.unige.it 
observed in human brains have a long history and many excellent results have been published, in the second contribution by Ruta and Gabrys an inspiration for artificial learning has been found in the mechanics of physical fields which can be observed on both micro and macro scales. The authors demonstrate how a simple concept of a physical field can be adopted to build a complete framework for supervised and unsupervised learning methodology. Exploiting interesting analogies between data and charged particles subjected to gravity, electrostatic and gas particle fields, a family of new algorithms has been developed and applied to classification, clustering and data condensation.

One of the most common and omnipresent tasks related to adaptation and learning are combinatorial optimization problems which have been subject of study of many researchers for a long time. In recent years and with the increased computational power of modern computing technology nature-inspired algorithms based on observation of foraging behaviour of ants or swarming behaviour of flocks of birds, to name just two of such inspirations, have emerged and proved to be highly competitive for many well known, difficult combinatorial optimization problems. In the third contribution Bianchi et al. provide a comprehensive account of such nature-inspired as well as other recently proposed algorithms, known under a collective name of metaheuristics, for solving optimization problems with uncertain, stochastic and dynamic information.

The final contribution in this issue is an example of another family of approaches which due to some theoretical advancements and outstanding practical performance have taken significant steps forward in recent years. It is concerned with learning algorithms in multiple classifier and prediction systems also known under many other names like committees of machines, ensemble methods, etc. In their contribution Minku et al. propose an approach to building neural network ensembles using negative correlation learning algorithm and extending it to the case of incremental learning so critical in dynamically changing environments. The principles of encouraging diversity among individuals and building collaborative teams so frequently observed in nature in the context of adaptation and survival are central to these approaches and frequently appearing in many other disciplines of science and engineering.

While the contributions in this special issue provide only a small glimpse into a very exciting and very quickly growing area of nature-inspired technologies with various applications to a large number of different domains requiring learning and adaptation features interested readers are further encouraged to visit the NiSIS Co-ordination Action project materials which are freely available or search using the following terms: Ant colonies; Artificial immune systems; Attention, perception and cognition models; Autonomic computing; Auditory/Vision models; Evolvable systems; Membrane computing; Molecular, cellular and DNA computing; Quorum computing; Self assembly; Swarm intelligence; Chemical and quantum computing; Physically inspired models; Artificial neural networks; Social phenomena based complex self-adaptive techniques. 